\title{
Myelin Oligodendrocyte Glycoprotein (MOG) Antibody Disease in a 11 Year Old with COVID-19 Infection
}

\author{
Archana Khan ${ }^{1}$ (D) $\cdot$ Hiren Panwala ${ }^{2}$ (D) Divya Ramadoss ${ }^{1}$ (D) $\cdot$ Raju Khubchandani $^{1}$ (D)
}

Received: 25 October 2020 / Accepted: 31 December 2020 / Published online: 20 January 2021

(C) Dr. K C Chaudhuri Foundation 2021

To the Editor: SARS-CoV-2 causes multiorgan damage. There is an increasing evidence of extra-pulmonary involvement including the nerve cells and eye [1]. MOG is a glycoprotein located on the myelin surface and optic neuritis is the commonest presentation of myelin oligodendrocyte glycoprotein antibody disease (MOGAD) seen in 54\%-61\% of patients [1]. There are two reports of SARS CoV-2 associated MOG-AD causing optic neuritis in a 15 and 26 y old [2, 3].

A 11-year-old boy presented with redness and ophthalmodynia in both eyes four days after a brief febrile illness. Two weeks later, there was overnight loss of vision in the right eye without any systemic or neurologic complaints. There was bilateral conjunctival congestion with painful movement of eye. Right eye findings showed normal perception and projection of light, sluggishly reactive pupils, vision of $<\mathrm{N} / 36$ and disc edema of Grade 3 relative afferent pupillary defect. Left eye vision was 6/9. Hemogram, erythrocyte sedimentation rate (ESR) and C-reactive protein (CRP) were normal. SARS-CoV-2 nasopharyngeal swab was positive by CBNAAT. Serum anti-SARS-CoV-2 immunoglobulin (IgG) antibodies were reactive 20.8 (Normal range $<1.0$ ). Serum MOG antibody was positive. Aquaporin4 antibody was not detected. Cerebrospinal fluid (CSF) showed mild lymphocytic pleocytosis $(55 / \mathrm{mL}$ normal $<5$ cells $/ \mathrm{mL}$ ) while proteins and glucose were normal. Oligoclonal bands, MOG-IgG and cultures were

Archana Khan arch.agr@gmail.com

1 Section of Pediatric Rheumatology, NH SRCC Children's Hospital, Mumbai, India

2 Department of Pediatric Radiology, NH SRCC Children's Hospital, Mumbai, India negative. CSF- SARS-CoV-2 ribonucleic acid (RNA) polymerase chain reaction (PCR) was not done.

Magnetic resonance imaging (MRI) findings showed bilateral asymmetrical optic neuritis, involving intraorbital and intracanalicular portion on the right and intraorbital portion on the left. Altered signal intensities were segmental and diffuse in the right intraorbital optic nerve and patchy in the left intraorbital optic nerve with enhancement of the optic nerve sheath in the right orbit. The chiasmatic, retrochiasmatic portion, optic tracts, rest of brain and spine were normal. These findings were consistent with MOG antibody associated disease [4]. Response to pulse methylprednisolone was dramatic. Oral steroids were continued and tapered over 12 wk. Right eye vision improved to $6 / 9$ and left eye to $6 / 6$ by day 10 .

We thus concluded a diagnosis of MOG-AD-associated bilateral optic neuritis adding to the small body of evidence of SARS-CoV-2 associated MOG-AD as yet another neurological feature of this deadly virus.

Acknowledgments Dr. Hitendra Mehta (Referring Ophthalmologist) diagnosed optic neuritis in this child.

\section{Compliance with Ethical Standards}

Consent and Declaration for Publication Fee A written informed consent was obtained from the family to publish this manuscript.

Conflict of Interest None.

\section{References}

1. Baig AM, Sanders EC. Potential neuroinvasive pathways of SARSCoV-2: deciphering the spectrum of neurological deficit seen in coronavirus disease-2019 (COVID-19). J Med Virol. 2020;92:184557.

2. de Ruijter NS, Kramer G, Gons RAR, Hengstman GJD. Neuromyelitis optica spectrum disorder after presumed coronavirus 
(COVID-19) infection: a case report. Mult Scler Relat Disord. 2020;46:102474.

3. Zhou S, Jones-Lopez EC, Soneji DJ, Azevedo CJ, Patel VR. Myelin oligodendrocyte glycoprotein antibody-associated optic neuritis and myelitis in COVID-19. J Neuroophthalmol. 2020;40(3):398-402.
4. Denève $\mathrm{M}$, Biotti $\mathrm{D}$, Patsoura $\mathrm{S}$, et al. MRI features of demyelinating disease associated with anti-MOG antibodies in adults. J Neuroradiol. 2019;46(5):312-8.

Publisher's Note Springer Nature remains neutral with regard to jurisdictional claims in published maps and institutional affiliations. 